\title{
Hydroxychloroquine screening in the UK-closing the gaps
}

\author{
Anne Gobbett $\mathbb{D}^{1}$
}

Received: 12 January 2021 / Revised: 12 January 2021 / Accepted: 14 January 2021 / Published online: 29 January 2021

(c) The Author(s), under exclusive licence to The Royal College of Ophthalmologists 2021

Hydroxychloroquine (HCQ) has caused some excitement over the past year due to its potential use as a treatment for or prophylaxis against Covid 19. It has also been a topic for much discussion in the Ophthalmology world following the publication of a paper by Melles and Marmor in 2014 [1] who, using spectral domain optical coherence tomography (SD-OCT) and wide field auto-fluorescence (AF), suggested that the rate of HCQ retinopathy was higher than previously thought $(7.5 \%)$.

Chloroquine and HCQ have been used in the treatment of inflammatory diseases for 70 years [2]. Reports of retinal toxicity in the early years of its use led to the development of various screening programmes [3]. During 1990 within the UK, it was decided that screening for HCQ retinopathy in the Hospital Eye Service (HES) was no longer necessary [4]. Further published papers suggested the rate of HCQ retinopathy was only $0.5-2.0 \%$ [5-7]. The work of Melles and Marmor [1] precipitated changes in screening guidelines in the USA in 2016 [8] and by the Royal College of Ophthalmologists (RCOphth) in 2018 [9]. These latter guidelines suggested baseline screening for all patients starting HCQ of colour fundus photography and macula SD-OCT. Those at risk, requiring annual screening (duration $>5$ years, dose $>5 \mathrm{mg} / \mathrm{kg} /$ day, estimated glomerular filtration rate $<60 \mathrm{ml} / \mathrm{min} / 1.73 \mathrm{~m}^{2}$ or concurrent use of Tamoxifen) requiring wide field AF images and 10:2 Humphrey visual field (HVF) testing in addition. Definite toxicity was defined as having two abnormal test results. Possible retinopathy was defined as having only one abnormal test result.

Anne Gobbett

agobbett@doctors.org.uk

1 Sunderland Eye Infirmary, Sunderland, UK

\section{The first gap: screening for HCQ retinopathy has not been made available throughout the UK}

Most Ophthalmology units in the UK are understaffed [10]. Ophthalmology has the highest out-patient throughput of all specialities with nearly 8 million appointments a year in England alone in 2018-2019 [11]. Up to 320,000 patients are currently prescribed HCQ in England [9]. Screening these patients would therefore have demanded an increase of up to $4 \%$ in out-patient workload. Due to insufficient resources many ophthalmology units were unable to provide a screening service at all or only deliver a partial service to those patients considered to be at higher risk (personal communications). No screening has been performed in Scotland. Eye Health Scotland states "The additional Ophthalmology capacity required to allow implementation can only be achieved to the detriment of the existing care of people with active sight-threatening conditions, who may permanently lose sight without timely intervention". (https://www.optometryscotland.org.uk/wp-content/uploads/ 2019/10/Position-StatementHydrochloroquine.pdf).

\section{The second gap: the recently published results of HCQ screening according to RCOphth guidelines [12, 13] are not consistent with the results found by Melles and Marmor [1]}

The work of Marshall et al. [12] suggested a rate of $1.6 \%$ for definite HCQ toxicity (two positive tests), whilst Gobbett et al. [13] found a rate of $0.8 \%$ for definite toxicity. These results are significantly lower than those of Melles and Marmor 7.5\% [1] $(p=0.0001)$ and are more in keeping with the older literature [5-7]. Another recent paper exploring the experience of 68 Lupus experts with a cohort of 46,000 patients between them revealed a prevalence of retinal toxicity of $1.05 \%$ [14]. 
Melles and Marmor's data however is not based on patients having two positive tests for HCQ retinopathy but only one, either HVF or SD-OCT. It is likely their figures include both definite and possible retinopathy as defined by RCOphth guidelines [9]. Including definite and possible retinopathy numbers from the recent UK studies, an additional $4.7 \%$ [12] and $0.8 \%$ [13], respectively, the results are comparable (adjusted for demographic differences between the cohorts). Is this "gap" of possible retinopathy between definite toxicity and no toxicity clinically important?

\section{Closing the gaps}

\section{Monitoring for HCQ retinopathy}

The RCOphth changed the wording of its HCQ "screening" guidelines to "monitoring" in January 2020 [15] implying that HES assessment of patients taking HCQ was not obligatory. More radical changes were made to these guidelines in December 2020 [16] based on recent literature review including the work of Marshall et al. [12] and Gobbett et al. [13]. Baseline monitoring has been removed, and annual monitoring for those at risk no longer requires HVF unless an abnormality has been found on SD-OCT or AF. These changes will result in considerable reductions in the resources, time and finance, needed to run a monitoring service. They may enable more units to provide appropriate monitoring.

\section{Possible HCQ retinopathy}

The RCOphth does not recommend stopping HCQ if a diagnosis of possible HCQ retinopathy is made. Is it of clinical importance to be able to detect the possible HCQ retinopathy?

Shared decision making and consent are fundamental to good medical practice. "Patients should be informed of the potential benefits, risks of harm, uncertainties about and likelihood of success of any treatment" (General Medical Council) [17]. Good practice therefore determines that if a diagnosis of possible retinopathy can be made, this information should be made known to both the patient and treating physician, and that we should continue to monitor for it. Decisions in the treatment of inflammatory and autoimmune conditions are complex, many treatments carrying significant side effects therefore all available information on individual risk is important.

\section{The future}

A retrospective pilot longitudinal study of 6 patients with at least 3 examinations over an average of 3.5 years preceding diagnosis of HCQ retinopathy has recently been published by Marmor [18]. The author reports "dramatic retinal thinning across the posterior pole beginning 4-5 years before clinical diagnosis, with parafoveal regions thinning even faster". A larger longitudinal study looking at serial SD-OCT images may well prove important in determining possible HCQ retinopathy leading to the possibility of developing software to identify this thinning automatically and may further modify our monitoring guidelines.

\section{Compliance with ethical standards}

Conflict of interest The author declares no conflict of interest.

Publisher's note Springer Nature remains neutral with regard to jurisdictional claims in published maps and institutional affiliations.

\section{References}

1. Melles RB, Marmor MF. The risk of toxic retinopathy in patients on long-term hydroxychloroquine therapy. JAMA Ophthalmol. 2014;132:1453-60.

2. Shee JC. Lupus erythematosus treated with chloroquine. Lancet. 1953;265:201-2.

3. Hobbs HE, Sorsby A, Freedman A. Retinopathy following chloroquine therapy. Lancet. 1958;2:478-80.

4. Morsman CDG, Livesey SJ, Richards IM, Jessop JD, Mills PV. Screening for hydroxychloroquine retinal toxicity: Is it necessary? Eye. 1990;4:572-6.

5. Wolfe F, Marmor MF. Rates and predictors of hydroxychloroquine retinal toxicity in patients with rheumatoid arthritis and systemic lupus erythematosus. Arthritis Care Res (Hoboken). 2010;62:775-84

6. Yam JC, Kwok AK. Ocular toxicity of hydroxychloroquine. Hong Kong Med J. 2006;12:294-304.

7. Spalton DJ, Verdon Roes GM, Hughes GRV. Hydroxychloroquine, dosage parameters and retinopathy. Lupus. 1993;2:355-8.

8. Marmor MF, Kellner U, Lai TY, Melles RB, Mieler WF, for the American Academy of Ophthalmology. Recommendations on screening for chloroquine and Hydroxychloroquine retinopathy (2016 Revision). Ophthalmology. 2016;123:1386-94.

9. Hydroxychloroquine and chloroquine retinopathy:recommendations on screening. RCOphth February 2018 https://www. rcophth.ac.uk/wpcontent/uploads/2018/07/Hydroxychloroquine-a nd-Chloroquine-Retinopathy-ScreeningGuideline-Recommenda tions.pdf

10. Hingorani M, Harcourt J. Workforce census 2018 RCOphth. https://www.rcophth.ac.uk/wp-content/uploads/2019/ 02/RCOphth-Workforce-Census-2018.pdf

11. Hospital Outpatient Activity 2018-19 National statistics Publication Date: 10 Oct 2019. https://digital.nhs.uk/data-andinformation/publications/statistical/hospital-outpatient-activity/ 2018-19

12. Marshall E, Robertson M, Kam S, Penwarden A, Riga P, Davies N. Prevalence of hydroxychloroquine retinopathy using 2018 Royal College of Ophthalmologists diagnostic criteria. Eye. 2021;35:343-8.

13. Gobbett A, Kotagiri A, Bracewell C, Smith J. Two years' experience of screening for hydroxychloroquine retinopathy. 
Eye. 2020. https://doi.org/10.1038/s41433-020-1028-4. Online ahead of print.

14. Winebrake J, Khalili L, Weiner J, et al. Rheumatologists' perspective on hydroxychloroquine guidelines. Lupus Sci Med. 2020;7:e000427. https://doi.org/10.1136/lupus-2020-000427

15. Hydroxychloroquine and Chloroquine Retinopathy: Recommendations on Monitoring. RCOphth. January $2020 \mathrm{https}: / / \mathrm{www}$. rcophth.ac.uk/wp-content/uploads/2020/02/HCR-Recommenda tions-on-Monitoring.pdf

16. Hydroxychloroquine and Chloroquine Retinopathy: Recommendations on Monitoring. RCOphth. December 2020 https://www.
rcophth.ac.uk/wp-content/uploads/2020/12/Hydroxychloroquine-a nd-Chloroquine-Retinopathy-Monitoring-Guideline.pdf

17. Decision making and consent. General Medical Council. November 2020. https://www.gmc-uk.org/ethical-guidance/ethica 1-guidance-for-doctors/decision-making-and-consent

18. Marmor MF, Durbin M, de Sisternes L, Pham BH. Sequential retinal thickness analysis shows hydroxychloroquine damage before other screening techniques. Retinal Cases \& Brief Reports: December 18, 2020 - Volume Publish Ahead of Print - Issue https://doi.org/10.1097/ICB.0000000000001108 Published: 23 September 2020 